\title{
Agonist-independent GPCR activity and receptor-instructed axonal projection in the mouse olfactory system
}

\author{
Hirofumi Nishizumi ${ }^{1}$ \& Hitoshi Sakano*,1 \\ ${ }^{1}$ Department of Brain Function, School of Medical Sciences, University of Fukui, Matsuoka, Fukui 910-1193, Japan \\ *Author for correspondence: sakano.hts@gmail.com \\ $\boldsymbol{~}$ it is the OR-derived receptor signals, rather than the direct action of OR molecules, that instruct \\ targeting of OSN axons"
}

First draft submitted: 3 June 2019; Accepted for publication: 16 October 2019; Published online:

16 December 2019

Keywords: axon targeting • baseline activity • CAMP • G-protein coupled receptors • olfactory sensory neurons • olfactory system • spontaneous structural transition

In mice, various odorants can be detected with approximately 1000 odorant receptor (OR) species expressed in olfactory sensory neurons (OSNs) present in the olfactory epithelium (OE). Each OSN expresses only one functional $O R$ gene not only in a mutually exclusive but also mono-allelic manner. Furthermore, OSN axons bearing the same OR species converge their axons to a specific pair of glomeruli at stereotyped locations in the olfactory bulb $(\mathrm{OB})$. Thus, the odor information detected in the $\mathrm{OE}$ is topographically represented in the OB as the pattern of activated glomeruli [1].

A remarkable feature of OSN projection is that OR molecules play instructive roles in projecting OSN axons to the OB. Interestingly, OR molecules can be detected in axon termini by using a fluorescent tag or by immunostaining. Based on these findings, it has been suggested that the OR protein itself may find guidance cues in the target and also mediate homophilic adhesive interactions of the same type of OSN axons. However, recent studies suggest that instead of functioning as axon guidance and sorting molecules, OR-specific receptor activity determines the transcription levels of axon-guidance and axon-sorting molecules using cyclic adenosine monophosphate (cAMP) as a second messenger [2].

During development, an olfactory map is generated by a combination of dorsal-ventral (D-V) targeting and anterior-posterior (A-P) targeting followed by activity-dependent map refinement. For D-V targeting projection, positional information of OSN cell bodies within the OE regulates the levels of D-V targeting molecules [3]. In contrast, A-P targeting is instructed by OR molecules. Spontaneous OR-activity without involving ligands regulates the transcription of A-P targeting molecules via cAMP whose levels are uniquely determined by the expressed OR species [4]. OR-derived cAMP also regulates the transcription levels of axon sorting molecules for glomerular segregation and olfactory map refinement [5]. However, unlike A-P targeting, glomerular segregation is regulated by the neuronal activity of OSNs. How do the OR molecules differentially regulate both A-P targeting and glomerular segregation using cAMP as a second messenger? What are the sources of cAMP, and how do the signals separately regulate these two processes? Recent studies have unveiled these questions by using mutant mice for axon guidance and signaling molecules.

\section{Projection along the A-P axis}

In mature OSNs, the binding of odorants to ORs is converted into a neuronal activity. The olfactory-specific $\mathrm{G}$ protein $\left(\mathrm{G}_{\mathrm{olf}}\right)$ mediates cAMP production by activating adenylyl cyclase type III. This cAMP opens cyclic nucleotide gated $(\mathrm{CNG})$ channels and depolarizes the plasma membrane, thus generating the action potential. Knocking-out $(\mathrm{KO})$ of $\mathrm{G}_{\text {olf }}$ and $\mathrm{CNG}-\mathrm{A} 2$, a component of CNG channels, causes severe anosmia. Despite their essential roles in odor signal transduction, these KOs do not demonstrate major defects in axonal projection of 
OSNs [6], although it was later found that the CNG-A2 KO affects the activity-dependent synapse formation and glomerular segregation [5]. Therefore, it was initially thought that OR-derived cAMP signals were not essential for OSN projection.

Although the KOs of $\mathrm{G}_{\text {olf }}$ and CNG-A2 do not affect axon targeting of OSNs, the KO of ADCY3 causes severe defects in glomerular map formation [7]. Thus, it was suggested that OR-instructed OSN projection uses a different $G$ protein and does not require neuronal activity. To examine this possibility, Imai et al. [2] generated a mutant OR whose G-protein coupling motif, Asp-Arg-Tyr (DRY), was mutated. The DRY motif is commonly found in the cytoplasmic end of transmembrane domain III of G-protein coupled receptors (GPCRs). With the DRY-motif mutant, OSN axons remain in the anterior OB and fail to converge to a specific glomerulus. These defects are restored by co-expression of the constitutively active (ca) mutant of stimulus $G$ protein, $G_{s}$. Partial rescue is also observed with the ca mutant of PKA. Thus, PKA-mediated noncanonical signaling pathway is involved in A-P projection, using $\mathrm{G}_{s}$. Interestingly, $\mathrm{caG}_{\mathrm{s}}$ results in a posterior shift of glomeruli when expressed with the wild-type (WT) OR, whereas dominant-negative PKA causes an anterior shift [2]. These findings demonstrate that it is the OR-derived receptor signals, rather than the direct action of OR molecules, that instruct targeting of OSN axons along the A-P axis.

In OSN axons, an axon-guidance molecule Neuropilin $1(\mathrm{Nrp1})$ is found in a graded manner, anteriorlow/posterior-high, in the OB. Increases and decreases of Nrp1 in OSNs result in posterior and anterior shifts of glomeruli, respectively [8]. How do the axon guidance molecules determine the topographic order of the olfactory map? Sperry [9] proposed the 'chemo-affinity model' for axonal projection, in which target cells express chemical cues to guide axons to their destination. However, in the mouse olfactory system, OSN axons are able to converge even in the absence of the OB. It has been reported that map order emerges in axon bundles, before they reach the target OB. Imai et al. [8] found that pretarget sorting of OSN axons plays an important role in organizing of the olfactory map. Nrp1 and its repulsive ligand Semaphorin 3A (Sema3A) are expressed in a complementary manner within the axon bundles of OSNs. Furthermore, $\mathrm{Nrp}^{\text {low }} / \mathrm{Sema} 3 \mathrm{~A}^{\text {high }}$ axons are sorted to the central compartment of the bundle, whereas $\mathrm{Nrp}^{\text {high }} / \mathrm{Sema} 3 \mathrm{~A}^{\text {low }}$ axons are confined to the outer lateral part of the bundle. OSN-specific conditional $\mathrm{KO}(\mathrm{cKO})$ of $\mathrm{Nrp} 1$ or Sema3A not only perturbs axon sorting within the bundle, but also causes shifts of glomeruli along the A-P axis. These results demonstrate that pretarget axon sorting within bundles contributes to olfactory map formation.

Recently, there are reports claiming that A-P targeting cannot be explained thoroughly by Nrp1/Sema3A signaling $[10,11]$. They report that ectopic glomeruli are found in the Nrp1 cKO rather than the anteriorly shifted glomerulus, challenging the paper by Imai et al. $[2,8]$. Although such ectopic glomeruli often appear in the Nrp1 $\mathrm{cKO}$, they tend to be found on the anterior side of the WT glomerulus. Multiple ectopic glomeruli are generally found in the KOs of targeting molecules. The reason why multiple glomeruli appear in the KOs is probably due to the looser convergence of OSN axons. For unknown reasons, the target area becomes more dispersed in the KOs than in the WT. As a result, these axons cannot converge to a single glomerular structure and thus generate multiple ectopic glomeruli with the same OR identity. In addition to the previous studies on $\mathrm{Nrp} 1[2,8]$, mutant analyses of $\beta 2$-adrenergic receptor ( $\beta 2-\mathrm{AR}$ ) [4] further support the involvement of $\mathrm{Nrp} 1$ in $\mathrm{A}-\mathrm{P}$ targeting. Thus, it is clear that Nrp1 plays an essential role in A-P targeting of OSN axons. However, it is still possible that additional sets of axon guidance molecules are involved in this process as suggested [10,11]. For example, Plexin A1 (PlxnA1) is distributed in a graded manner, anterior-high and posterior-low, whose transcription is also, but inversely, regulated by OR-derived cAMP $[2,3]$.

\section{Projection of OSNs along the $\mathrm{D}-\mathrm{V}$ axis}

For OSN targeting along the $\mathrm{D}-\mathrm{V}$ axis, there is a close correlation between the locations of OSN cell bodies in the $\mathrm{OE}$ and their target sites in the $\mathrm{OB}$. The preservation of spatial relationships of neuronal cell bodies and their projection sites is seen in other sensory systems [12]. Two sets of repulsive signaling molecules, Robo2/Slit1 and $\mathrm{Nrp} 2 /$ Sema3F, are known to be involved in $\mathrm{D}-\mathrm{V}$ targeting in the mouse olfactory system $[3,13]$. These guidance molecules contribute to the separation of dorsal (D) and ventral (V) OB domains.

How is the positional information of OSNs in the OE translated to their target site during glomerular map formation? An axon-guidance receptor $\mathrm{Nrp} 2$ is expressed in OSNs in a graded manner along the D-V axis. It was initially thought that the repulsive ligand Sema3F was produced in the $\mathrm{OB}$ as a target cue showing a counter gradient along the D-V axis. However, the Sema3F transcript was not detected in the OB cells. As found for A-P 
targeting molecules, Nrp1 and Sema3A [8], Nrp2 and Sema3F are both expressed in OSN axons in a complementary manner to regulate D-V targeting [3].

It was found that during development, OSNs in the $\mathrm{D}$ region mature earlier than those in the $\mathrm{V}$ region $\mathrm{OE}$. The D-region OSNs target their axons to the embryonic OB before the $\mathrm{V}$-region axons arrive. Early arriving D-region axons secrete a repulsive ligand Sema3F in the anterodorsal $\mathrm{OB}$ to repel late-arriving $\mathrm{Nrp} 2^{+} \mathrm{V}$-region axons, thus separating $\mathrm{D}$ and $\mathrm{V}$ region axons [3]. Then, what kind of molecules guide pioneer OSN axons to the anterodorsal area acting as a landmark in the $\mathrm{OB}$ ? Robo $2^{+} \mathrm{D}$-region axons are guided to the $\mathrm{D}$ domain of the $\mathrm{OB}$ by repulsive interactions with a secreted ligand for Robo2, Slit1. During development, the glomerular map expands ventrally and axonal projection of OSNs occurs sequentially from the $\mathrm{D}$ to the $\mathrm{V}$ region in the $\mathrm{OB}$. This sequential arrival of OSN axons helps establish the glomerular order along the D-V axis.

\section{Activity-dependent glomerular segregation \& map refinement}

During development, an olfactory map is established based on a genetic program independently from the neuronal activity. After OSN axons reach their destinations, further refinement of the olfactory map needs to occur through fasciculation of OSN axons. To study how OR-specific glomerular segregation is regulated, a group of genes were searched for, whose expression profiles are correlated with the OR species [5]. Two sets of axon-sorting molecules were identified in OSN axons: one set was homophilic adhesive molecules, Kirrel2 and Kirrel3, and the other set was repulsive molecules, ephrin-A5 ligand and EphA5 receptor. A specific set of axon-sorting molecules, whose expression levels are uniquely determined by the OR identity, regulate the fasciculation of OSN axons. Repulsive interactions between the two sets of axons, ephrin-A $5^{\text {high }} / \mathrm{EphA} 5^{\text {low }}$ and ephrin-A $5^{\text {low }} / \mathrm{EphA} 5^{\text {high }}$, are important for the separation of dissimilar axons. Homophilic interactions between the two sets of axons, Kirrel $2^{\text {high }} /$ Kirrel $3^{\text {low }}$ and Kirrel2 $2^{\text {low }} /$ Kirrel $3^{\text {high }}$, mediate bundling of similar axons.

Recently, using OSNs in ex vivo acute slices of the neonatal OE, Nakashima et al. [14] reported that expression levels of axon sorting molecules may be determined by OR-specific spike-patterns of spontaneous OSN activity [14]. It will be interesting to study how the temporal firing pattern is read out by OSNs and utilized for differential regulation of axon sorting molecules. An olfactory map generated during development is initially a continuous map whose topography is established by complimentary distributions of axon guidance receptors and their repulsive ligands. The map is then converted to a discrete glomerular map after birth. Activity-dependent axon sorting contributes not only to the glomerular map refinement, but also to the conversion of the map from continuous to discrete [15].

\section{Agonist-independent OR activities}

As mentioned above, OR-derived signals for global targeting are not stimulus driven. Then, what kind of OR activity could be responsible for A-P targeting? Crystallographic analyses revealed that GPCRs possess two distinct conformations, active and inactive [15]. In the absence of agonists and inverse agonists, GPCRs generate basal levels of cAMP by spontaneously flipping between the two conformational states.

For different OR species, variable but specific levels of baseline activities can be detected in the absence of ligands [4]. This agonist-independent GPCR activity had long been considered to be noise, and its functional role was not fully appreciated. As naris occlusion does not affect targeting of OSN axons, the OR activity that regulates A-P projection appears to be ligand independent. To examine this, various mutants of the $\beta 2$-AR have proved useful. The $\beta 2-A R$ is a GPCR with the highest sequence similarity to ORs (10-20\% amino acid identity). When expressed with the $O R$ gene promoter, the $\beta 2$-AR follows the one neuron-one receptor rule and substitutes ORs for OR-instructed axon targeting of OSNs [16]. The key amino-acid residues are well characterized in the $\beta 2-A R$, for example, G-protein coupling and ligand binding. Furthermore, the 3D structures are known for $\beta 2$-AR with or without $G_{s}$ [17]. As a result of these favorable features, Nakashima et al. [4] analyzed $\beta 2-A R$ mutants for the agonist-independent GPCR activity.

Among the mutants, those that affect conformational transitions demonstrate altered agonist-independent activity. In the transgenic mice expressing both mutant and WT $\beta 2-\mathrm{AR}$, the activity-low mutants generate glomeruli anterior to those of the WT, whereas the activity-high mutations cause a posterior shift of glomeruli [4]. Nrp1 levels are elevated in OSNs expressing the activity-high $\beta 2$-AR mutants, but are lowered by the activity-low mutations. The results are inverse for another A-P targeting molecule, PlxnA1, because its expression is inversely regulated by cAMP. It is notable that levels of glomerular segregation molecules are not affected by the baseline-activity mutations. 
It has long been puzzling how the OR molecules instruct axonal projection of OSNs to the OB. It is now clear that the agonist-independent baseline-activity is responsible for OR-instructed axonal projection. It is not clear at this point whether the functional usage of baseline activity is something general for GPCRs or specific for ORs. It will be interesting to study in the future, whether the baseline activity is also utilized in other GPCR systems as found for ORs in the mouse olfactory system. Another long-standing question was differential regulation of A-P targeting and glomerular segregation that are both regulated by OR-derived cAMP signals. How are these two types of regulation separately controlled in OSNs? During olfactory development, $\mathrm{G}_{s}$ is expressed in immature OSNs whereas $\mathrm{G}_{\text {olf }}$ is expressed in mature OSNs. KO studies indicate that A-P targeting and glomerular segregation are separately regulated by $\mathrm{G}_{\mathrm{s}}$ and $\mathrm{G}_{\mathrm{olf}}$, respectively, at different stages of OSNs development [4].

\section{Activity-dependent synapse formation}

It was initially thought that precise circuit connections are not affected in the $\mathrm{KO}$ of $\mathrm{CNG}$ channels [6]. Although targeting of OSN axons is not affected in the CNG-A2 KO, activity-dependent processes, for example, glomerular segregation and synapse formation, are perturbed. How are the OSN axons connected firmly with dendrites of mitral/tufted (M/T) cells by the OR-derived OSN activity? What kind of signaling is essential for inducing the postsynaptic events? To address these questions, Inoue et al. [18] searched for a receptor and ligand pair, expressed either in OSN axons or in M/T-cell dendrites in the neonatal glomeruli. Among almost 30 axon guidance, cell adhesion and signaling molecules examined, Sema7A and its receptor PlxnC1 turned to be promising.

Sema7A is a membrane protein localized to the presynaptic axon-terminal of OSNs. Sema7A expression is promoted by odor stimulation, but is abolished in the CNG-channel KO. Unlike other Sema-family proteins, for example, Sema3A and Sema3F, targeting of OSN axons is not affected in the KO for Sema7A. However, synapse formation and dendrite selection are perturbed in the M/T cells. Furthermore, in the Sema7A KO, synaptic markers are markedly reduced and postsynaptic density is rarely found in $\mathrm{M} / \mathrm{T}$-cell dendrites. PlxnC1 is a receptor for Sema7A and is localized to the dendrites of M/T cells only in neonates. In contrast to Sema7A, expression of PlxnC1 is not affected by odor stimuli, but its localization to the M/T-cell dendrites is limited to the first week after birth. In the M/T-specific $\mathrm{CKO}$ of PlxnC1, postsynaptic events are perturbed and postsynaptic density formation is blocked as observed in the Sema7A KO. Since both Sema7A and PlxnC1 are expressed in the membrane-bound form, they can directly interact with each other and function as signaling molecules to induce the post-synaptic events in $\mathrm{M} / \mathrm{T}$ cells [18].

\section{Summary \& discussion}

In the mammalian olfactory system, a glomerular map is generated using specific sets of axon guidance and sorting molecules expressed in OSN axons. A characteristic feature of OSN projection is that olfactory map formation is instructed by OR molecules. It has long been puzzling how the axon targeting and axon sorting are differentially instructed by the same OR identity both using cAMP as a second messenger. Recent studies revealed that glomerular segregation is regulated by the stimulus-driven OR activity via CNG channels, whereas A-P targeting is instructed by the ligand-independent OR activity via KA. Furthermore, these two processes are separately regulated by using different $G$ proteins, $G_{s}$ and $G_{\text {olf }}$, at different stages of OSN maturation.

For A-P targeting, the OR activity that regulates axon guidance molecules is not odor evoked. How is this activity generated for cAMP production without odor ligands? In the absence of agonists and inverse agonists, GPCRs freely interchange their conformations between the active and inactive forms. Baseline activity generated by spontaneous transitions is utilized for A-P targeting in immature OSNs. It was once thought that spontaneous neuronal activity might be responsible for regulating OSN projection. However, this hypothesis is not supported by the blocking experiments of OSN activity using the Kir2.1 overexpression mouse [4,15]. As for the spontaneous activity, Lorenzon et al. [19] found that spontaneous firing is needed for glomerular segregation and intra-bulbar connections using the Kir2.1 mouse. More recently, Nakashima et al. [14] demonstrated that temporal spike patterns of spontaneous firing may regulate OR-specific differential expression of glomerular segregation molecules. It will be interesting to study how the spike patterns of spontaneous activity contribute to segregation of OSN axons and circuit connections within the OB.

For D-V targeting, the early-arriving D-region axons secrete Sema3F that ventrally repels the late-arriving Nrp2 ${ }^{+}$ $\mathrm{V}$-region axons, thus establishing the topographic order of glomeruli along the $\mathrm{D}-\mathrm{V}$ axis [3]. Like in the glomerular layer for OSN axons, Nrp2 levels are high in the $\mathrm{V}$ region, but low in the $\mathrm{D}$ region in the mitral-cell layer. Recently, Inokuchi et al. [20] found that $\mathrm{Nrp}^{+} \mathrm{MCs}$ migrate to the $\mathrm{V}$-region $\mathrm{OB}$ as the $\mathrm{OB}$ ventrally expands, whereas 
$\mathrm{Nrp2} 2^{-} \mathrm{MCs}$ remain in the embryonic $\mathrm{OB}$ that represents the D-region OB in adults. Sema3F secreted by the Dregion OSNs guides both $\mathrm{Nrp}^{+}$OSN axons and $\mathrm{Nrp}^{+} \mathrm{MCs}$ to the posteroventral $\mathrm{OB}$ [20]. This co-regulation is important for proper matching between the glomeruli and MC primary dendrites. Since MC dendrites synapse with OSN axons in the nearest neighboring glomeruli [21], D/V partitioning provides a topographical and functional separation of MCs within the OB.

In mice, olfactory circuits for innate responses are hard wired and are generated based on a genetic program. However, in neonates, the circuits can be modulated by the odor-evoked OSN activity in an OR-specific manner. After birth, there is a narrow time window referred to as the critical period that allows proper development of the system in response to environmental inputs. In the mouse olfactory system, a pair of signaling molecules, Sema7A and its receptor PlxnC1, is sufficient for inducing postsynaptic events in the neonatal M/T cells [18]. Within the Sema7A-stimulated glomeruli, primary dendrites of surrounding $M / T$ cells are recruited, thus, enlarging the glomerular sizes. Elevated odor inputs through the enlarged glomeruli appear to be responsible for establishing imprinted neonatal memory that induces positive responses to the imprinted odorants as adults.

In the mouse olfactory system, basic processes of primary projection from the $\mathrm{OE}$ to $\mathrm{OB}$ have now been elucidated. However, secondary projection from the OB to the olfactory cortex is largely unknown. This is mainly due to the absence of known subset markers for M/T cells. It is hoped that such markers will be found and their cKO studies will shed light on the molecular basis of decision making for odor-induced behaviors. The mouse olfactory system will continue to serve as an excellent model system for our understanding of the mammalian brain.

\section{Financial \& competing interests disclosure}

This work was supported by JSPS KAKENHI Grants (JP24000014 and JP17H06160 to H Sakano and JP19H04745 to H Nishizumi). The authors have no other relevant affiliations or financial involvement with any organization or entity with a financial interest in or financial conflict with the subject matter or materials discussed in the manuscript apart from those disclosed.

No writing assistance was utilized in the production of this manuscript.

\section{References}

1. Mori K, Sakano H. How is the olfactory map formed and interpreted in the mammalian brain? Annu. Rev. Neurosci. 34, 467-499 (2011).

2. Imai T, Suzuki M, Sakano H. Odorant receptor-derived cAMP signals direct axonal targeting. Science 314(5799), 657-661 (2006).

3. Takeuchi H, Inokuchi K, Aoki M et al. Sequential arrival and graded secretion of Sema3F by olfactory neuron axons specify map topography at the bulb. Cell 141(6), 1056-1067 (2010).

4. Nakashima A, Takeuchi H, Imai T et al. Agonist-independent GPCR activity regulates axon targeting of olfactory sensory neurons. Cell 154(6), 1314-1325 (2013).

5. Serizawa S, Miyamichi K, Takeuchi $\mathrm{H}$ et al. A neuronal identity code for the odorant receptor-specific and activity-dependent axon sorting. Cell 127(5), 1057-1069 (2006).

6. Lin DM, Wang F, Lowe G et al. Formation of precise connections in the olfactory bulb occurs in the absence of odorant-evoked neuronal activity. Neuron 26(1), 69-80 (2000).

7. Col JA, Matsuo T, Storm DR et al. Adenylyl cyclase-dependent axonal targeting in the olfactory system. Development 134(13), 2481-2489 (2007).

8. Imai T, Yamazaki T, Kobayakawa R et al. Pre-target axon sorting establishes the neural map topography. Science 325(5940), 585-590 (2009).

9. Sperry RW. Chemoaffinity in the orderly growth of nerve fiber patterns and connections. Proc. Natl Acad. Sci. USA 50, 703-710 (1963).

10. Assens A, Dal Col JA, Njoku A et al. Alteration of Nrp1 signaling at different stages of olfactory neuron maturation promotes glomerular shifts along distinct axes in the olfactory bulb. Development 143(20), 3817-3825 (2016).

11. Zapiec B, Bressel OC, Khan M et al. Neuropilin-1 and the positions of glomeruli in the mouse olfactory bulb. eNeuro 3(5), e0123-16 (2016).

12. Luo L, Flanagan JG. Development of continuous and discrete neural maps. Neuron 56(2), 284-300 (2007).

13. Aoki M, Takeuchi H, Nakashima A et al. Possible roles of robo $1^{+}$ensheathing cells in guiding dorsal-zone olfactory sensory neurons in mouse. Dev. Neurobiol. 73(11), 828-840 (2013).

14. Nakashima A, Ihara N, Shigeta M et al. Structured spike series specify gene expression patterns for olfactory circuit formation. Science 365(6448), eaaw5030 (2019).

15. Yu CR, Power J, Barnea G et al. Spontaneous neural activity is required for the establishment and maintenance of the olfactory sensory map. Neuron 42(4), 553-566 (2004). 
16. Rasmussen SG, DeVree BT, Zou Y et al. Crystal structure of the $\beta 2$ adrenergic receptor-Gs protein complex. Nature 477(7366), 549-555 (2011).

17. Feinstein P, Bozza T, Rodriguez I et al. Axon guidance of mouse olfactory sensory neurons by odorant receptors and the $\beta 2$ adrenergic receptor. Cell 117(6), 833-846 (2004).

18. Inoue $\mathrm{N}$, Nishizumi $\mathrm{H}$, Naritsuka $\mathrm{H}$ et al. Sema7A/PlxnCl signaling triggers activity-dependent olfactory synapse formation. Nat. Commun. 9, 1842 (2018).

19. Lorenzon P, Redolfi N, Podolsky MJ et al. Circuit formation and function in the olfactory bulb of mice with reduced spontaneous afferent activity. J. Neurosci. 35(1), 146-160 (2015).

20. Inokuchi K, Imamura F, Takeuchi $\mathrm{H}$ et al. $\mathrm{Nrp} 2$ is sufficient to instruct circuit formation of mitral-cells to mediate odour-induced attractive social responses. Nat. Commun. 8, 15977 (2017).

21. Nishizumi H, Miyashita A, Inoue N et al. Primary dendrites of mitral cells synapse unto neighboring glomeruli independent of their odorant receptor identity. Commun. Biol. 2, 14 (2019). 